\title{
DUALITY IN SUB-SUPERCRITICAL BUBBLING IN THE BREZIS-NIRENBERG PROBLEM NEAR THE CRITICAL EXPONENT
}

\author{
MANUEL DEL PINO, JEAN DOLBEAULT, AND MONICA MUSSO
}

\section{INTRODUCTION}

Let $\Omega \subset \mathbb{R}^{3}$ be a bounded domain with smooth boundary and consider the boundary value problem

$$
\begin{cases}\Delta u+\lambda u+u^{q}=0 & \text { in } \Omega, \\ u>0 & \text { in } \Omega, \\ u=0 & \text { on } \partial \Omega .\end{cases}
$$

Integrating the equation against a first eigenfunction of the Laplacian yields that a necessary condition for solvability of (1.1) is $\lambda<\lambda_{1}$. On the other hand, if $1<q<5$ and $0<\lambda<\lambda_{1}$, a solution may be found as follows. Let us consider the Rayleigh quotient

$$
Q_{\lambda}(u):=\frac{\int_{\Omega}|\nabla u|^{2}-\lambda \int_{\Omega}|u|^{2}}{\left(\int_{\Omega}|u|^{q+1}\right)^{\frac{2}{q+1}}} \quad \text { for any } u \in H_{0}^{1}(\Omega) \backslash\{0\}
$$

and set

$$
S_{\lambda}:=\inf _{u \in H_{0}^{1}(\Omega) \backslash\{0\}} Q_{\lambda}(u) .
$$

The constant $S_{\lambda}$ is achieved thanks to the compactness of Sobolev's embedding if $q<5$, and a suitable scalar multiple of it turns out to be a solution of (1.1). The case $q \geq 5$ is considerably more delicate: for $q=5$ compactness of the embedding is lost while for $q>5$ there is no such embedding. This obstruction is not just technical for the solvability question, but essential. Pohozaev [11] showed that if $\Omega$ is strictly star-shaped then no solution of (1.1) exists if $\lambda \leq 0$ and $q \geq 5$. Let $S_{0}$ be the best constant in the critical Sobolev's embedding,

$$
S_{0}=\inf _{u \in C_{0}^{1}\left(\mathbb{R}^{3}\right) \backslash\{0\}} \frac{\int_{\mathbb{R}^{3}}|\nabla u|^{2}}{\left(\int_{\mathbb{R}^{3}}|u|^{6}\right)^{1 / 3}} .
$$

Let us consider first the case $q=5$ in (1.2) and define the number

$$
\lambda^{*}=\inf \left\{\lambda>0: S_{\lambda}<S_{0}\right\} .
$$

In a well-known paper, [2], Brezis and Nirenberg established that $0<\lambda^{*}<$ $\lambda_{1}$ and, as a consequence, that $S_{\lambda}$ is achieved for $\lambda^{*}<\lambda<\lambda_{1}$, hence (1.1) 
is solvable in this range. When $\Omega$ is a ball they find that $\lambda^{*}=\lambda_{1} / 4$, while no solution exists for $\lambda \leq \lambda^{*}$.

Let us assume now that $q>5$. In this case Sobolev's embedding fails and the quantity $S_{\lambda}$ may only be interpreted as zero. Thus, no direct variational approach applies to find existence of solutions. Consequences of the analysis of this paper are the following existence and multiplicity results for Problem (1.1) in the super-critical regime when $q$ is sufficiently close to 5 .

Theorem 1. (a) Assume that $\lambda^{*}<\lambda<\lambda_{1}$, where $\lambda^{*}$ is the number given by (1.5). Then there exists a number $q_{1}>5$ such that Problem (1.1) is solvable for any $q \in\left(5, q_{1}\right)$.

(b) Assume that $\Omega$ is a ball and that $\lambda_{1} / 4<\lambda<\lambda_{1}$. Then, given $k \geq 1$ there exists a number $q_{k}>5$ such that Problem (1.1) has at least $k$ radial solutions for any $q \in\left(5, q_{k}\right)$.

While the result of Part (a) resembles that by Brezis and Nirenberg when $q=5$, in reality the solution we find has a very different nature: it blows up as $q \downarrow 5$ developing a single bubble around certain point inside the domain. The other solutions predicted by Part (b) blow-up only at the origin but exhibit multiple bubbling. Let us make this terminology somewhat more precise. By a blowing-up solution for (1.1) near the critical exponent we mean an unbounded sequence of solutions $u_{n}$ of (1.1) for $\lambda=\lambda_{n}$ bounded, and $q=q_{n} \rightarrow 5$. Setting

$$
M_{n}=\alpha^{-1} \max _{\Omega} u_{n}=\alpha^{-1} u_{n}\left(x_{n}\right) \rightarrow+\infty
$$

with $\alpha>0$ to be chosen, we see then that the scaled function

$$
v_{n}(y)=M_{n}^{-1} u_{n}\left(x_{n}+M_{n}^{\left(q_{n}-1\right) / 2} y\right)
$$

satisfies

$$
\Delta v_{n}+v_{n}^{q_{n}}+M_{n}^{-\left(q_{n}-1\right)} \lambda_{n} v_{n}=0
$$

in the expanding domain $\Omega_{n}=M_{n}^{\left(q_{n}-1\right) / 2}\left(\Omega-x_{n}\right)$. Assuming for instance that $x_{n}$ stays away from the boundary of $\Omega$, elliptic regularity implies that locally over compacts around the origin, $v_{n}$ converges up to subsequences to a positive solution of

$$
\Delta w+w^{5}=0
$$

in entire space, with $w(0)=\max w=\alpha$. It is well-known, see for instance [4] that for the convenient choice $\alpha=3^{1 / 4}$, this solution is explicitly given by

$$
w(z)=\frac{3^{1 / 4}}{\sqrt{1+|z|^{2}}}
$$

which corresponds precisely to an extremal of the Sobolev constant $S_{0}$. Coming back to the original variable, we expect that "near $x_{n}$ " the behavior of $u_{n}(y)$ can be approximated as

$$
u_{n}(y)=\frac{3^{1 / 4} M_{n}}{\sqrt{1+M_{n}^{4}\left|y-x_{n}\right|^{2}}}(1+o(1)) .
$$


Since the convergence in expanded variables is only local over compacts, it is not clear how far from $x_{n}$ the approximation (1.7) holds true, even if only one maximum point $x_{n}$ exists. We say that the solution $u_{n}(x)$ is a single bubble if (1.7) holds with $o(1) \rightarrow 0$ uniformly in $\Omega$. The solution predicted by Part (a) has this property around a point of the domain that will be precised below, while those of Part (b) have the form of a "tower" of single bubbles centered at the origin. As we shall see, radial symmetry is not needed for the presence of these solutions: just symmetry with respect to the three coordinate planes around one point of the domain suffices. The

results of [3] concerning asymptotic analysis of radial solutions in a ball when the exponent approaches the critical exponent from below, suggest that the object ruling the location of blowing-up in single-bubble solutions of (1.1) is Robin's function $g_{\lambda}$ defined as follows. Let $\lambda<\lambda_{1}$ and consider Green's function $G_{\lambda}(x, y)$, solution for given $x \in \Omega$ of

$$
\begin{array}{rlrl}
-\Delta_{y} G_{\lambda}-\lambda G_{\lambda} & =\delta_{x} & y \in \Omega, \\
G_{\lambda}(x, y) & =0 & y \in \partial \Omega .
\end{array}
$$

Then we define

$$
g_{\lambda}(x):=H_{\lambda}(x, x)
$$

where

$$
H_{\lambda}(x, y):=\frac{1}{4 \pi|y-x|}-G_{\lambda}(x, y) .
$$

It turns out that $g_{\lambda}(x)$ is a smooth function which goes to $+\infty$ as $x$ approaches $\partial \Omega$. Its minimum value is not necessarily positive. In fact this number is decreasing in $\lambda$. It is strictly positive when $\lambda$ is close to 0 and approaches $-\infty$ as $\lambda \uparrow \lambda_{1}$. It is suggested in [3] and recently proven by Druet in [9] that the number $\lambda^{*}$ given by (1.5) can be characterized as

$$
\lambda^{*}=\sup \left\{\lambda>0: \min _{\Omega} g_{\lambda}>0\right\} .
$$

Besides, it is shown in [9] that least energy solutions $u_{\lambda}$ for $\lambda \downarrow \lambda^{*}$ constitute a single-bubble with blowing-up near the set where $g_{\lambda_{*}}$ attains its minimum value zero.

We consider here the role of non-trivial critical values of $g_{\lambda}$ in existence of solutions of (1.1) in dimension $N=3$. In fact their role is intimate, not only in the critical case $q=5$ and in the sub-critical $q=5-\varepsilon$. More interesting, their connection with solvability of (1.1) for powers above critical is found. In fact phenomena apparently unknown even in the case of the ball is established, which put in evidence an amusing duality between the sub and super-critical cases.

The meaning we give of a non-trivial critical value of $g_{\lambda}$ is as follows: Let $\mathcal{D}$ be an open subset of $\Omega$ with smooth boundary. We recall that $g_{\lambda}$ links non-trivially in $\mathcal{D}$ at critical level $\mathcal{G}_{\lambda}$ relative to $B$ and $B_{0}$ if $B$ and 
$B_{0}$ are closed subsets of $\overline{\mathcal{D}}$ with $B$ connected and $B_{0} \subset B$ such that the following conditions hold: if we set $\Gamma=\left\{\Phi \in C(B, \mathcal{D}) /\left.\Phi\right|_{B_{0}}=\operatorname{Id}\right\}$, then

$$
\sup _{y \in B_{0}} g_{\lambda}(y)<\mathcal{G}_{\lambda}:=\inf _{\Phi \in \Gamma} \sup _{y \in B} g_{\lambda}(\Phi(y))
$$

and for all $y \in \partial \mathcal{D}$ such that $g_{\lambda}(y)=\mathcal{G}_{\lambda}$, there exists a vector $\tau_{y}$ tangent to $\partial \mathcal{D}$ at $y$ such that

$$
\nabla g_{\lambda}(y) \cdot \tau_{y} \neq 0
$$

Under these conditions a critical point $\bar{y} \in \mathcal{D}$ of $g_{\lambda}$ with $g_{\lambda}(\bar{y})=\mathcal{G}_{\lambda}$ in fact exists. It is easy to check that the above conditions hold if

$$
\inf _{x \in \mathcal{D}} g_{\lambda}(x)<\inf _{x \in \partial \mathcal{D}} g_{\lambda}(x) \quad \text { or } \quad \sup _{x \in \mathcal{D}} g_{\lambda}(x)>\sup _{x \in \partial \mathcal{D}} g_{\lambda}(x),
$$

namely the case of (possibly degenerate) local minimum or maximum points of $g_{\lambda}$. The critical value $\mathcal{G}_{\lambda}$ may be taken in these cases respectively as that of the minimum and the maximum of $g_{\lambda}$ in $\mathcal{D}$. These also hold if $g_{\lambda}$ is $C^{1}$-close to a function with a nondegenerate critical point in $\mathcal{D}$. We call $\mathcal{G}_{\lambda}$ a non-trivial critical level of $g_{\lambda}$ in $\mathcal{D}$. This notion of local notrivial critical level was used in analyzing concentration phenomena in a different context in [5].

Theorem 2. Let us assume that there is a set $\mathcal{D}$ where $g_{\lambda}$ has a non-trivial critical level $\mathcal{G}_{\lambda}$.

(a) Assume that $\mathcal{G}_{\lambda}<0, q=5+\varepsilon$. Then Problem (1.1) is solvable for all sufficiently small $\varepsilon>0$. More precisely, there exists a solution $u_{\varepsilon}$ of (1.1) of the form

$$
u_{\varepsilon}(y)=\frac{3^{1 / 4} M_{\varepsilon}}{\sqrt{1+M_{\varepsilon}^{4}\left|y-\zeta_{\varepsilon}\right|^{2}}}(1+o(1))
$$

where $o(1) \rightarrow 0$ uniformly in $\bar{\Omega}$ as $\varepsilon \rightarrow 0$,

$$
M_{\varepsilon}=8 \sqrt{2\left(-\mathcal{G}_{\lambda}\right) \varepsilon^{-1}},
$$

and $\zeta_{\varepsilon} \in \mathcal{D}$ is such that $g_{\lambda}\left(\zeta_{\varepsilon}\right) \rightarrow \mathcal{G}_{\lambda}, \quad \nabla g_{\lambda}\left(\zeta_{\varepsilon}\right) \rightarrow 0$ as $\varepsilon \rightarrow 0$.

(b) Assume that $\mathcal{G}_{\lambda}>0, \quad q=5-\varepsilon$. Then Problem (1.1) has a solution $u_{\varepsilon}$ of (1.1) exactly as in Part (a) but with $M_{\varepsilon}=8 \sqrt{2 \mathcal{G}_{\lambda} \varepsilon^{-1}}$.

We observe then that Theorem 1 follows from Part (a) of the above result making use of the characterization (1.8) of the number $\lambda^{*}$. The result of Part (b) recovers the asymptotics found for the radial solution of (1.1) when $\Omega$ is a ball and $0<\lambda<\lambda_{1} / 4$ in Theorem 1 of [3].

Next we state our result concerning the presence of solutions with multiple bubbling from above the critical exponent in a domain exhibiting symmetries, from which Theorem 1 Part (b) is an immediate consequence. We say that $\Omega \subset \mathbb{R}^{3}$ is symmetric with respect to the coordinate planes if for all $\left(y_{1}, y_{2}, y_{3}\right) \in \Omega$ we have that

$$
\left(-y_{1}, y_{2}, y_{3}\right),\left(y_{1},-y_{2}, y_{3}\right),\left(y_{1}, y_{2},-y_{3}\right) \in \Omega \text {. }
$$


Theorem 3. Assume that $0 \in \Omega$, and that $\Omega$ is symmetric with respect to the coordinate axes. Assume also that $g_{\lambda}(0)<0$ and $q=5+\varepsilon$. Then, given $k \geq 1$, there exists for all sufficiently small $\varepsilon>0$ a solution $u_{\varepsilon}$ of Problem (1.1) of the form

$$
u_{\varepsilon}(x)=\sum_{j=1}^{k} \frac{3^{1 / 4} M_{j \varepsilon}}{\sqrt{1+M_{j \varepsilon}^{4}|x|^{2}}}(1+o(1))
$$

where $o(1) \rightarrow 0$ uniformly in $\bar{\Omega}$ and for $j=1, \ldots, k$,

$$
M_{j \varepsilon} \equiv 8 \sqrt{2\left(-g_{\lambda}(0)\right) k^{-1}}\left(\frac{32 \sqrt{2}}{\pi}\right)^{j-1} \frac{(k-j) !}{(k-1) !} \varepsilon^{\frac{1}{2}-j} .
$$

The solution predicted by this theorem is a superposition of $k$ bubbles with respective blow-up orders $\varepsilon^{\frac{1}{2}-j}$ for $j=1, \ldots, k$.

Bubbling solutions for semilinear equations near the critical exponent has been the object of various works in the literature. In particular we refer the reader to $[1,7,6,10,12]$ and references therein for construction of bubbling solutions in relation to Green's function of the domain. The results above do have analogues for dimension $N \geq 4$, which we shall state in the last section. It should be remarked that when $N \geq 4$ we have that $\lambda^{*}=0$. The object responsable for concentration and single-bubbling as $\lambda \downarrow 0$ or $\lambda=0$ and $q$ approaches critical from below, around a non-degenerate critical point of the function $g_{0}$, was first established in [12]. The phenomenon of multi-bubbling in the radial case in higher dimensions seems to have been observed first in [7]. A functional analytic setting that allowed to establish the presence of bubbling solutions for slightly supercritical powers and $\lambda=0$ in the presence of a small hole in the domain was devised in [6].

Next section will be devoted to sketch the proofs of the above results, whose full details will be provided in the forthcoming paper [8]. In the last section we discuss how these results extend to higher dimensions.

\section{Sketch of Proofs}

Let $\zeta$ be a point in $\Omega$. We consider spherical coordinates $y=y(\rho, \theta)$ centered at $\zeta$ given by

$$
\rho=|y-\zeta| \text { and } \theta=\frac{y-\zeta}{|y-\zeta|} .
$$

We consider now the transformation transformation

$$
v(x, \theta)=\mathcal{T}_{q}(u)(x, \theta):=2^{\frac{2}{q-1}} e^{-x} u\left(\zeta+e^{-2 x} y\right) .
$$

Let us denote by $D$ the $\zeta$-dependent subset of $S=\mathbb{R} \times S^{2}$ where the variables $(x, \theta)$ vary. After these changes of variables, problem (1.1) becomes

$$
\begin{gathered}
4 \Delta_{S^{2}} v+v^{\prime \prime}-v+4 \lambda e^{-4 x} v+e^{(q-5) x} v^{q}=0 \quad \text { in } D \\
v>0 \quad \text { in } D, \quad v=0 \quad \text { on } \partial D .
\end{gathered}
$$


Here and in what follows, ${ }^{\prime}=\frac{\partial}{\partial x}$. Let us write

$$
w_{\mu, \zeta}(y) \equiv \frac{3^{1 / 4}}{\sqrt{1+\mu^{-2}|y-\zeta|^{2}}} \mu^{-\frac{1}{2}},
$$

for a point $\zeta \in \mathbb{R}^{3}$ and a positive number $\mu$. We observe that

$$
\mathcal{T}_{5} w_{\mu, \zeta}(x, \theta)=W(x-\xi),
$$

where $W(x)=(12)^{1 / 4} e^{-x}\left(1+e^{-4 x}\right)^{-1 / 2}$ and $\mu=e^{-2 \xi}$. $W$ is the unique solution of the problem

$$
\left\{\begin{array}{l}
W^{\prime \prime}-W+W^{5}=0 \quad \text { on }(-\infty, \infty) \\
W^{\prime}(0)=0 \\
W>0, \quad W(x) \rightarrow 0 \text { as } x \rightarrow \pm \infty
\end{array}\right.
$$

Let us consider points $0<\xi_{1}<\xi_{2}<\cdots<\xi_{k}$. We look for a solution of $(2.2)$ of the form

$$
v(x, \theta)=\sum_{i=1}^{k}\left(W\left(x-\xi_{i}\right)+\Pi_{i}(x, \theta)\right)+\phi(x, \theta)
$$

where $\phi$ is small and $\Pi_{i}(x, \theta)$ satisfies the boundary value problem

$$
\begin{array}{ll}
-\left(4 \Delta_{S^{2}} \Pi+\Pi^{\prime \prime}-\Pi+4 \lambda e^{-4 x} \Pi\right)=4 \lambda e^{-4 x} W(x-\xi) & \text { in } D, \\
\Pi=-W(x-\xi) & \text { on } \partial D .
\end{array}
$$

A main observation is that $v(x, \theta) \sim \sum_{i=1}^{k} W\left(x-\xi_{i}\right)$ solves (2.2) if and only if, going back in the change of variables, with $\mu_{i}=e^{-2 \xi_{i}}$,

$$
u(y) \sim \mathcal{T}_{q}^{-1}\left(\sum_{i=1}^{k} W\left(x-\xi_{i}\right)\right) \sim \sum_{i=1}^{k} \frac{3^{1 / 4} \mu_{i}^{-1 / 2}}{\sqrt{1+\mu_{i}^{-2}|y-\zeta|^{2}}},
$$

solves (1.1). Let us write

$$
W_{i}(x):=W\left(x-\xi_{i}\right), \quad V_{i}:=W_{i}+\Pi_{i}, \quad V:=\sum_{i=1}^{k} V_{i} .
$$

If we set $v:=V+\phi$. then solving (2.2) is equivalent to finding $\phi$ such that

$$
\begin{cases}L_{q, \lambda} \phi=-N_{q}(\phi)-R_{q} & \text { in } D \\ \phi=0 & \text { on } \partial D\end{cases}
$$

where, with $c_{q} \equiv 2^{-(q-5) / 2}$,

$$
\begin{aligned}
& L_{q, \lambda}(\phi):=4 \Delta_{S^{2}} \phi+\phi^{\prime \prime}-\phi+4 \lambda e^{-4 x} \phi+q c_{q} e^{(q-5) x} V^{q-1} \phi, \\
& N(\phi):=c_{q} e^{(q-5) x}\left[(V+\phi)_{+}^{q}-V^{q}-q V^{q-1} \phi\right],
\end{aligned}
$$


and

$$
R_{q}:=c_{q} e^{(q-5) x} V^{q}-\sum_{i=1}^{k} W_{i}^{5} .
$$

Rather than solving (2.2) directly, we consider first the following intermediate problem: Given points $\xi=\left(\xi_{1}, \ldots, \xi_{k}\right) \in \mathbb{R}^{k}$ and a point $\zeta \in \Omega$, find a function $\phi$ such that for certain constants $c_{i j}$,

$$
\left\{\begin{array}{l}
L_{q, \lambda} \phi=-N_{q}(\phi)-R_{q}+\sum c_{i j} Z_{i j} \text { in } D \\
\phi=0 \text { on } \partial D, \\
\int_{D} Z_{i j} \phi d x d \theta=0 \text { for all } i, j,
\end{array}\right.
$$

where $Z_{i j}(x, \theta)=\mathcal{T}_{5}\left(z_{i j}\right), i=1, \ldots, k, j=1, \ldots, N+1$, with $z_{i j}$ is the solution of the boundary value problem

$$
\begin{cases}-\left(\Delta z_{i j}+\lambda z_{i j}\right)=-\Delta \hat{z}_{i j} & \text { in } \Omega \\ z_{i j}=0 & \text { on } \partial \Omega\end{cases}
$$

and the $\hat{z}_{i j}$ are respectively given for $i=1, \ldots, k, j=1, \ldots, N$, by

$$
\hat{z}_{i j}(y)=\frac{\partial}{\partial \zeta_{j}} w_{\mu_{i}, \zeta}(y), \quad \hat{z}_{(N+1) j}(y)=\frac{\partial}{\partial \mu_{i}} w_{\mu_{i}, \zeta}(y) .
$$

A straightforward computation then yields that $Z_{i j}$ satisfies

$$
\begin{cases}-\left(4 \Delta_{S^{2}} Z_{i j}+Z_{i j}^{\prime \prime}-Z_{i j}+4 \lambda e^{-4 x} Z_{i j}\right)=5 W_{i}^{4} \hat{Z}_{i j} & \text { in } D \\ Z_{i j}=0 & \text { on } \partial D\end{cases}
$$

with $\hat{Z}_{i j}=\mathcal{T}_{5}\left(\hat{z}_{i j}\right)$. We will see that problem (2.9) is uniquely solvable if the points $\xi_{i}, \zeta$ satisfy appropriate constraints and $q$ is close enough to 5 . To this end we consider first the linear problem in $(\bar{D})$ : Find $\phi$ such that for certain real numbers $c_{i j}$

$$
\begin{cases}L_{q, \lambda} \phi=h+\sum c_{i j} Z_{i j} & \text { in } D, \\ \phi=0 & \text { on } \partial D, \\ \int_{D} Z_{i j} \phi=0 & \forall i, j .\end{cases}
$$

We need uniformly bounded solvability in proper functional spaces for Problem (2.13), for a proper range of the $\xi_{i}$ 's and $\zeta$. To this end, it is convenient to introduce the following norm. Given a small but fixed positive number $\sigma$ let us set us set

$$
\|\psi\|_{*}:=\sup _{(x, \theta) \in D} \nu^{-1}(x)|\psi(x, \theta)| \quad \text { with } \quad \nu(x):=e^{-x}+\sum_{i=1}^{k} e^{-\sigma\left|x-\xi_{i}\right|}
$$

and consider the subspace $\mathcal{C}_{*}$ of the continuous functions $\psi$ for which $\|\psi\|_{*}$ is finite. Let $\varepsilon=|q-5|$. Then we have the following result. 
Lemma 1. Let $\lambda \in\left(0, \lambda_{1}-\delta\right)$ and $\operatorname{dist}(\zeta, \partial \Omega)>\delta$ for some small positive $\delta$.

There exist positive numbers $\varepsilon_{0}, \delta_{0}, R_{0}$, and a constant $C>0$ such that if the points $0<\xi_{1}<\xi_{2}<\cdots<\xi_{k}$ satisfy

$$
R_{0}<\xi_{1}, \quad R_{0}<\min _{1 \leq i<k}\left(\xi_{i+1}-\xi_{i}\right), \quad \xi_{k}<\frac{\delta_{0}}{\varepsilon_{0}} \quad \text { if } q \neq 5, \quad \xi_{k}<\infty,
$$

then for all $|q-5|<\varepsilon_{0}$ and all $h \in C(D)$ with $\|h\|_{*}<+\infty$, Problem (2.9) admits a unique solution $\phi=: T_{q, \lambda}(h)$. Besides,

$$
\left\|T_{q, \lambda}(h)\right\|_{*} \leq C\|h\|_{*} \quad \text { and } \quad\left|c_{i j}\right| \leq C\|h\|_{*} .
$$

Consider the map $\xi \mapsto T_{\varepsilon}$, with values on $\mathcal{L}\left(\mathcal{C}_{*}\right)$. This map is of class $C^{1}$. Moreover, there is a constant $C>0$ such that

$$
\left\|D_{\xi} T_{\varepsilon}\right\|_{\mathcal{L}\left(\mathcal{C}_{*}\right)} \leq C
$$

uniformly on $\xi$ and $\lambda$ satisfying conditions (2.15).

To solve Problem (2.9) we restrict conveniently the range of the parameters $\xi_{i}$. Let us consider for a number $M$ large but fixed, the following conditions:

$$
\left\{\begin{array}{c}
\xi_{1}>\frac{1}{2} \log (M \varepsilon)^{-1}, \quad \log (M \varepsilon)^{-1}<\min _{1 \leq i<k}\left(\xi_{i+1}-\xi_{i}\right), \\
\xi_{k}<k \log (M \varepsilon)^{-1}
\end{array}\right.
$$

Useful facts that we easily check is that under relations (2.16), $N_{q}$ and $R_{q}$ defined by (2.8), (2.8) satisfy for all small $\varepsilon>0$ and $\|\phi\|_{*} \leq 1 / 4$ the estimates:

$$
\left\|N_{q}(\phi)\right\|_{*} \leq C\|\phi\|_{*}^{2} \quad \text { and } \quad\left\|R^{q}\right\|_{*} \leq C(\varepsilon|\log \varepsilon|+|q-5|),
$$

provided $\varepsilon=|q-5|$ is small.

Lemma 2. Let $\sigma<1$. Assume that relations (2.16) hold and let $\varepsilon=$ $|q-5|$.Then there is a constant $C>0$ such that, for all $\varepsilon \geq 0$ small enough, there exists a unique solution $\phi=\phi\left(\xi_{1}, \ldots, \xi_{k}, \zeta\right)=\phi(\bar{\xi}, \zeta)$ to problem (2.9) which besides satisfies

$$
\|\phi\|_{*} \leq C \varepsilon^{1-\sigma} \quad \text { and } \quad\left\|D_{\xi, \zeta} \phi\right\|_{*} \leq C \varepsilon^{1-\sigma} .
$$

For the proof of the existence part we observe that $\phi$ solves (2.9) if and only if

$$
\phi=T_{q, \lambda}\left(N_{q}(\phi)+R_{q}\right)=: A_{q, \lambda}(\phi) .
$$

Thus we need to prove that the operator $A_{q, \lambda}$ defined above is a contraction in a proper region. Let us consider the set

$$
\mathcal{F}_{r}=\left\{\phi \in C(D):\|\phi\|_{*} \leq r \varepsilon^{1-\sigma}\right\}
$$

with $r$ a positive number to be fixed later. From Lemma 1 and (2.17), we get

$$
\left\|A_{q, \lambda}(\phi)\right\|_{*} \leq C\left\|N_{q}(\phi)+R_{q}\right\|_{*} \leq C\left[(r \varepsilon)^{2(1-\sigma)}+\varepsilon^{1-\sigma}\right]<r \varepsilon^{1-\sigma}
$$


for all small $\varepsilon$, provided that $r$ is chosen large enough, but independent of $\varepsilon$. Thus $A_{q, \lambda}$ maps $\mathcal{F}_{r}$ into itself for this choice of $r$. Moreover, $A_{q, \lambda}$ turns out to be a contraction mapping in this region. It is indeed easy to check that $N_{q}$ defines a contraction in the $\|\cdot\|_{*}$-norm.

According to the above result, our problem has been reduced to that of finding points $\xi_{i}$ and $\zeta$ so that the constants $c_{i j}$ which appear in (2.9), for the solution $\phi$ given by Lemma 2, are all zero. Thus we need to solve the system of equations

$$
c_{i j}(\bar{\xi}, \zeta)=0 \quad \text { for all } i, j .
$$

If (2.18) holds, then $v=V+\phi$ will be a solution to (2.2). This system turns out to be equivalent to a variational problem, which we introduce next.

Let us consider the functional

$$
\mathcal{F}_{q, \lambda}(\bar{\xi}, \zeta):=F_{q, \lambda}(V+\phi),
$$

where $\phi=\phi(\bar{\xi}, \zeta)$ is given by Lemma 2 and $F_{q, \lambda}$ is defined by

$$
\begin{aligned}
F_{q, \lambda}(v)= & 2 \int_{D}\left|\nabla_{\theta} v\right|^{2}+\frac{1}{2} \int_{D}\left[\left|v^{\prime}\right|^{2}+|v|^{2}\right] \\
& -2 \lambda \int_{D} e^{-4 x} v^{2}-\frac{1}{q+1} \int_{D} e^{(q-5) x}|v|^{q+1} .
\end{aligned}
$$

Solving system (2.18) turns out to be equivalent to finding a critical point of this functional, namely equivalent to

$$
\nabla \mathcal{F}_{q, \lambda}(\bar{\xi}, \zeta)=0 .
$$

The key point to find the desired solutions is the following fact, which is a consequence of the estimates given in Lemma 2.

Lemma 3. The following expansion holds

$$
\mathcal{F}_{q, \lambda}(\bar{\xi}, \zeta)=F_{q, \lambda}(V)+o(\varepsilon)
$$

where the term $o(\varepsilon)$ is uniform in the $C^{1}$-sense over all points satisfying constraint (2.16), for given $M>0$.

This result basically says that to obtain a solution of the problem, we need to find a critical point situation for the function $f_{q, \lambda}(\bar{\xi}, \zeta):=F_{q, \lambda}(V)$ which remains under $o(\varepsilon) C^{1}$ perturbations. It is convenient to relabel the $\xi_{i}$ 's in this functional, so that we define

$$
e_{q, \lambda}(\bar{\mu}, \zeta):=f_{q, \lambda}\left(-\frac{1}{2} \log \mu_{1}, \ldots,-\frac{1}{2} \log \mu_{k}, \zeta\right) .
$$

A straightforward computation yields that

$$
e_{q, \lambda}(\mu, \zeta)=E_{q, \lambda}(U)
$$

where

$$
U(y)=\sum_{j}\left[w_{\mu_{j}, \zeta}(y)+\pi_{\mu_{j}, \zeta}(y)\right],
$$


with $\pi_{\mu_{j}, \zeta}=\mathcal{T}_{5}^{-1}\left(\Pi_{\xi_{j}, \zeta}\right)$ and $\xi_{j}=-\frac{1}{2} \log \mu_{j}$, and for any $u: \Omega \rightarrow \mathbb{R}$,

$$
E_{q, \lambda}(u)=\frac{1}{2} \int_{\Omega}|D u|^{2}-\frac{\lambda}{2} \int_{\Omega} u^{2}-\frac{1}{q+1} \int_{\Omega} u^{q+1} .
$$

Next Lemma gives the energy estimate that shows that this critical point situation for the above functional indeed is present under the given assumptions.

Lemma 4. Assume that $\varepsilon=|q-5|$ and $\lambda \in\left(0, \lambda_{1}\right)$ such that $g_{\lambda}(\zeta) \neq 0$. Choose the parameters $\mu_{j}$ as follows

$$
\mu_{1}=\varepsilon \Lambda_{1}, \quad \mu_{j+1}=\mu_{j}\left(\Lambda_{j+1} \varepsilon\right)^{2}, \quad j=1, \ldots, k-1,
$$

for some positive numbers $\delta<\Lambda_{j}<\delta^{-1}$.

Then the following expansion holds,

$$
\begin{aligned}
e_{q, \lambda}(\mu, \zeta) & =k a_{0}+\frac{1}{2} k(k+1) a_{4} \varepsilon|\log \varepsilon|+o(\varepsilon) \\
+\varepsilon & \left\{a_{1} g_{\lambda}(\zeta) \Lambda_{1}+k a_{4} \log \Lambda_{1}+\sum_{j=2}^{k}\left[(k-j+1) a_{4} \log \Lambda_{j}-a_{5} \Lambda_{j}\right]\right\}
\end{aligned}
$$

uniformly with respect to the parameters $\Lambda_{j}$, as $\varepsilon \rightarrow 0$. Here $a_{i}$ 's are universal constants and the quantity $o(\varepsilon)$ is uniform in and $C^{1}$ senses in $(\zeta, \Lambda)$ for $\zeta$ ranging on compact sets.

The proof of this lemma is a rather lengthy computation which uses as a main factor the relationship between the term $\pi_{\mu_{j}, \zeta}$ and the regular part of Green's function $H_{\lambda}$, or more precisely, the fact that

$$
\pi_{\mu_{j}, \zeta}(y) \sim \mu^{1 / 2} H_{\lambda}(\zeta, y) .
$$

We recall that $g_{\lambda}(\zeta)=H_{\lambda}(\zeta, \zeta)$, and this is why this term appears in the expansion of the energy. With the above ingredients we are in a position to provide a proof of our main results.

Proof of Theorem 2. Let $q=5+\varepsilon$ and $\lambda \in\left(0, \lambda_{1}\right)$. We need to find a critical point of $\mathcal{E}(\mu, \zeta)=\mathcal{F}_{q, \lambda}\left(-\frac{1}{2} \log \mu, \zeta\right)$. We consider the change of variables $\mu=\mu(\Lambda)$ given by $\mu=\Lambda \varepsilon$, with $\Lambda>0$. Thus we are interested in finding critical points of the function $\psi_{\varepsilon}(\Lambda, \zeta)=\mathcal{E}(\mu(\Lambda), \zeta)$.

With the previous change of variables, the result in Lemma 3 and the expansion given in Lemma 4 read now as

$$
\psi_{\varepsilon}(\Lambda, \zeta)=a_{0}+\left[a_{1} g_{\lambda}(\zeta) \Lambda+a_{4} \log \Lambda\right] \varepsilon+a_{4} \varepsilon|\log \varepsilon|+o(\varepsilon)
$$

uniformly with respect to $\Lambda>\delta$ and $\operatorname{dist}(\zeta, \partial \Omega)>\delta$.

Again from Lemma 3, which actually holds with the $o(\varepsilon)$ term in the $C^{1}$ sense uniformly on $\Lambda>\delta$ and $\operatorname{dist}(\zeta, \partial \Omega)>\delta$, we obtain

$$
\nabla \psi_{\varepsilon}(\Lambda, \zeta)=\varepsilon[\nabla \psi(\Lambda, \zeta)+o(1)],
$$


where $\psi(\Lambda, \zeta)=a_{1} g_{\lambda}(\zeta) \Lambda+a_{4} \log \Lambda$. Let $D$ be the region where the assumed nontrivial linking situation for $g_{\lambda}$ holds. With no loss of generality we may assume that $g_{\lambda}(\zeta) \leq-\delta$ on $D$. We observe that for each fixed $\zeta$ the above function has a nondegenerate maximum, $\Lambda=\Lambda(\zeta)=-\frac{a_{4}}{a_{1} g_{\lambda}(\zeta)}$. Thus we get a critical point of $\psi$ if we find one of the function of $\zeta$

$$
\psi(\Lambda(\zeta), \zeta)=a_{0}+a_{4}\left[-1+\log \left(-\frac{a_{4}}{a_{1} g_{\lambda}(\zeta)}\right)\right] .
$$

The assumed linking structure for $g_{\lambda}$ in $D$ gives the presence of such a critical point. Using this and relation $(2.25)$ it is not hard to construct a region $\tilde{D}$ in the $(\Lambda, \zeta)$ variables where a nontrivial linking structure for the functional $\psi_{\varepsilon}(\Lambda, \zeta)$ is present, and which yields in $\zeta$ variable a point for which the value of $g_{\lambda}$ in close to $\mathcal{G}_{\lambda}$ and its gradient is close to zero. This finally yields the result of Part (a) of the Theorem.

As for Part $(b)$, we use the same argument as before, except that now $q=5-\varepsilon, \zeta_{\lambda}$ is a critical point for $g_{\lambda}$ with positive non-trivial critical value and

$$
\psi(\Lambda, \zeta)=a_{1} g_{\lambda}(\zeta) \Lambda-a_{4} \log \Lambda .
$$

Observe that in such a case, a nondegenerate minimum in $\Lambda$ variable is present, making it possible to argue symmetrically.

Proof of Theorem 3. Due to the symmetry of $\Omega$, the constants $c_{i j}$ that appear in (2.9) are automatically zero for any $j=1, \ldots, N$. We then just need to prove that the $c_{i(N+1)}$ 's are zero, for $i=1, \ldots, k$. Arguing again as in the proof of Theorem 2, we find a solution to problem (1.1) of the form (1.9) after we observe that the function

$$
\psi(\Lambda)=a_{1} g_{\lambda}(0) \Lambda_{1}+k a_{4} \log \Lambda_{1}+\sum_{j=2}^{k}\left[(k-j+1) a_{4} \log \Lambda_{j}-a_{5} \Lambda_{j}\right]
$$

given by (2.24) in Lemma 4, for the particular change of variable (2.23), has a nondegenerate critical point $\left(\Lambda_{1}, \ldots, \Lambda_{k}\right) \in \mathbb{R}_{+}^{k}$, which of course presists under small $C^{1}$-perturbations, thanks to the assumption $g_{\lambda}(0)<0$.

\section{HigheR DIMENSIONS}

In this section we state extensions of the results obtained in dimension $N=3$, for the super-critical case. The main difference between $N=3$ and $N \geq 4$ is that in the latter case the object ruling the single-bubbling phenomenon is the function $g_{0}$ (which is always positive), and no longer $g_{\lambda}$.

Let $N \geq 4$ and $\Omega \subset \mathbb{R}^{N}$ be a bounded smooth domain. We consider the problem

$$
\begin{cases}\Delta u+\lambda u+u^{\frac{N+2}{N-2}+\varepsilon}=0 & \text { in } \Omega \\ u>0 & \text { in } \Omega \\ u=0 & \text { on } \partial \Omega\end{cases}
$$


for some positive $\varepsilon$. The two results below yield in particular the validity of Theorem 1 for dimensions $N \geq 4$, where the numbers $\lambda^{*}$ and $\lambda_{1} / 4$ are now replaced by zero.

Theorem 4. Assume $N \geq 4$ and let us assume that there is a set $\mathcal{D}$ where $g_{0}$ has a non-trivial critical level $\mathcal{G}$. Assume also that $0<\lambda<\lambda_{1}$. Then, there exist points $\zeta_{\varepsilon}$ in $\mathcal{D}$, with $g_{0}\left(\zeta_{\varepsilon}\right) \rightarrow \mathcal{G}, \nabla g_{0}\left(\zeta_{\varepsilon}\right) \rightarrow 0$ and a solution to Problem (1.1) of the form

$$
u_{\varepsilon}(y)=\alpha_{N}\left(\frac{1}{1+\left(M_{\varepsilon}\right)^{\frac{4}{N-2}}|y-\zeta|^{2}}\right)^{\frac{N-2}{2}} M_{\varepsilon}(1+o(1)),
$$

with $o(1) \rightarrow 0$ uniformly on $\Omega$ as $\varepsilon \rightarrow 0$. In (3.2), $\alpha_{N}=(N(N-2))^{\frac{N-2}{4}}$ and

$$
\begin{aligned}
& M_{\varepsilon}=\beta \varepsilon^{-\frac{N-2}{4}} \quad \text { for } N \geq 5, \\
& M_{\varepsilon}=\beta \varepsilon^{-\frac{1}{2}}|\log \varepsilon|^{\frac{1}{2}} \quad \text { for } N=4,
\end{aligned}
$$

where $\beta$ is a positive (explicit) number which depends only on $\lambda, N$ and $\mathcal{G}$.

The corresponding result of multi-bubbling under symmetries extends in exactly the same way, now with the whole range $0<\lambda<\lambda_{1}$ allowed.

Theorem 5. Assume $N \geq 4,0 \in \Omega$ and suppose that $\Omega$ is symmetric with respect to the coordinate planes. Assume also that $0<\lambda<\lambda_{1}$. Then, given an integer $k \geq 1$, there exists a solution to Problem (3.1) of the form

$$
u_{\varepsilon}(y)=\alpha_{N} \sum_{j=1}^{k}\left(\frac{1}{1+\left(M_{j \varepsilon}\right)^{\frac{4}{N-2}}|y|^{2}}\right)^{\frac{N-2}{2}} M_{j \varepsilon}(1+o(1)),
$$

with $o(1) \rightarrow 0$ uniformly on $\Omega$ as $\varepsilon \rightarrow 0$. Here

$$
\begin{aligned}
& M_{j \varepsilon}=\beta_{j} \varepsilon^{-\frac{N-4}{2}-(j-1)} \quad \text { for } N \geq 5, \\
& M_{j \varepsilon}=\beta_{j} \varepsilon^{-\frac{1}{2}-(j-1)}|\log \varepsilon|^{\frac{1}{2}} \quad \text { for } N=4,
\end{aligned}
$$

where $\beta_{j}>0$ is an explicit number depending on $j, k, N, \lambda$ and $g_{0}(0)$.

\section{REFERENCES}

[1] A. BAHRI, Y. LI, O. REY, On a variational problem with lack of compactness: the topological effect of the critical points at infinity, Calc. of Var. 3 (1995), 67-93.

[2] H. Brezis, L. Nirenberg, Positive solutions of nonlinear elliptic equations involving critical Sobolev exponents, Comm. Pure Appl. Math. 36 no. 4 (1983), 437-477.

[3] H. Brezis, L.A. Peletier, Asymptotics for elliptic equations involving critical growth, Partial differential equations and the calculus of variations, Vol. I, 149192, Progr. Nonlinear Differential Equations Appl., 1, Birkhäuser Boston, 1989.

[4] L.A. Caffarelli, B. Gidas, J. Spruck, Asymptotic symmetry and local behavior of semilinear elliptic equations with critical Sobolev growth, Comm. Pure Appl. Math. 42 no. 3 (1989), 271-297.

[5] M. DEL Pino, P. Felmer, Semi-classical states for nonlinear Schrödinger equations, J. Funct. Anal. 149 no. 1 (1997), 245-265. 
[6] M. Del Pino, P. Felmer, M. Musso, Two-bubble solutions in the super-critical Bahri-Coron's problem, Calc. Var. Partial Diff. Eq. 16 no. 2 (2003), 113-145.

[7] M. Del Pino, J. Dolbeault, M. Musso, "Bubble-tower" radial solutions in the slightly supercritical Brezis-Nirenberg problem, J. Diff. Eq. 193 no. 2 (2003), 261543.

[8] M. Del Pino, J. Dolbeault, M. Musso, The Brezis-Nirenberg problem near criticality in dimension 3, Preprint.

[9] O. Druet, Elliptic equations with critical Sobolev exponents in dimension 3, Ann. Inst. H. Poincaré Anal. Non Linéaire 19 no. 2 (2002), 125-142.

[10] Z.-C. HAN, Asymptotic approach to singular solutions for nonlinear elliptic equations involving critical Sobolev exponent, Ann. Inst. H. Poincaré Anal. Non Linéaire 8 no. 2 (1991), 159-174.

[11] S. Pohozaev, Eigenfunctions of the equation $\Delta u+\lambda f(u)=0$, Soviet. Math. Dokl. 6, (1965), 1408-1411.

[12] O. REY, The role of the Green's function in a nonlinear elliptic equation involving the critical Sobolev exponent, J. Funct. Anal. 89 no. 1 (1990), 1-52.

M. Del Pino - Departamento de Ingeniería Matemática and CMM, Universidad de Chile, Casilla 170 Correo 3, Santiago, Chile.

J. Dolbeault - Ceremade (UMr CnRS no. 7534), Université Paris IX-Dauphine, Place de Lattre de Tassigny - 75775 Paris Cédex 16, France

M. Musso - Dipartimento di Matematica, Politecnico di Torino, Corso Duca DEgli Abruzzi, 24 - 10129 Torino, ItAly 